\title{
Sticky fingers
}

DOI:

10.1038/nrmicro1450

URLs

ONLINE LINKS

Entrez Gene: http://www.ncbi.

nlm.nih.gov/entrez/query.

fcgi?db=gene

gbs 1474

http://www.ncbi.nlm.nih.gov/ entrez/query.fcgi?db=gene\&c $\mathrm{md}=$ Retrieve\&dopt=full reportElist_uids $=1030548$

gbs 1477

http://www.ncbi.nlm.nih.gov/ entrez/query.fcgi?db=gene\&c $\mathrm{md}=$ Retrieve\&dopt=full reportElist_uids $=1030552$

gbs 1478

http://www.ncbi.nlm.nih.gov/ entrez/query.fcgi?db=gene\&c $\mathrm{md}=$ Retrieve\&dopt=full report\&list_uids=1030551

gbs1479

http://www.ncbi.nlm.nih.gov/ entrez/query.fcgi?db=gene\&c $\mathrm{md}=$ RetrieveEdopt=full reportElist_uids $=1030555$

Entrez Genome Project: http:// www.ncbi.nlm.nih.gov/entrez/ query.fcgi?db=genomeprj

\section{NEM316}

http://www.ncbi.nlm.nih.gov/ entrez/query.fcgi?db=genome prj\&cmd=Retrieve\&dopt $=$ Ove view\&list_uids=334

UniProtKB: http://ca.expasy. org/sprot

\section{Gbs1474}

http://www.expasy.org/ uniprot/Q8E4C7

\section{Gbs1477}

http://www.expasy.org/ uniprot/Q8E4C4

\section{Gbs1478}

http://www.expasy.org/

uniprot/Q8E4C3

\section{$\operatorname{RogB}$}

http://www.expasy.org/ uniprot/Q936E3
Although the pili of Gram-negative bacteria have been well described, only a few Gram-positive pathogens have been shown to express pili. Shaynoor Dramsi, Patrick Trieu-Cuot and colleagues now describe the function and mechanism of assembly of the pili of the Gram-positive pathogen Group B Streptococcus (GBS).

There are many reasons to study pilus elaboration in GBS. Analysis of the complete genomes of three GBS strains has identified loci that encode known components of pilus biosynthesis. These include surfaceanchored proteins that contain the LPXTG motif and putative sortases - enzymes that anchor LPXTG proteins to the bacterial cell wall. Also, a recent study by John Telford and colleagues at Novartis Vaccines showed that the SrtC locus of one GBS strain encodes proteins that assemble into pili, but the function and mechanism of assembly of these appendages were unknown.

To further investigate the role of the SrtC locus in pilin biosynthesis, Dramsi and colleagues performed a structural and functional analysis of the sortase loci in the GBS strain NEM316. Similar to other sequenced strains of GBS, the NEM316 strain has five sortase genes, one encoding a class A sortase and four genes encoding class $\mathrm{C}$ sortases. The genes that encode the class $\mathrm{C}$ sortases are tandemly arranged in two loci srtC1-C2 and srtC3-C4. In the NEM316 strain, the srtC3-C4 locus consists of the two sortase genes, $\operatorname{srtC} 3$ and srtC4 flanked by genes encoding the structural LPXTG surface proteins ( $g b s 1474, g b s 1477$ and $g b s 1478$ ), and includes an upstream regulatory gene gbs1479 (previously known as $\operatorname{rog} B$ ). The srtC1-C2 locus has a similar genetic organization, but in the NEM316 strain, only the srtC3-C4 locus is expressed at high levels.

The authors used immunogold electron microscopy (EM) of wildtype and mutant GBS NEM316 strains to confirm that the three structural proteins (Gbs1474, Gbs1477 and Gbs1478) encoded by the srtC3-C4 locus polymerize to form pilus-like structures. Gbs1477 is the major pilus component and is evenly distributed along the pilus core. Pilus-like structures were absent in the gbs 1477 mutant strain. Gbs1474 and Gbs1478 are minor pilin-associated proteins, which are not essential for pilus formation. These proteins are unevenly distributed along the pilus-like structure. Both Gbs1474 and Gbs1478 are found at the base of the pilus, and Gbs1478 also accumulates at the tip.

Immunogold EM and RT-PCR analyses of mutant GBS strains revealed insights into the regulation of pilus biosynthesis in GBS. SrtA is required for the accumulation of $g b s 1477, g b s 1478$ and $g b s 1474$ transcripts, although whether SrtA functions at the level of transcription or by stabilizing mRNA remains unclear. In addition, these studies confirmed that $\operatorname{Rog} B$ is a transcriptional activator of the $s r t C 3-C 4$ operon.

Finally, human pulmonary epithelial cell adherence assays revealed that Gbs1478 functions as a pilus-associated adhesin, providing compelling evidence that pili have a functional role in host-pathogen interactions in Gram-positive pathogens.

Shannon Amoils

ORIGINAL RESEARCH PAPER Dramsi, S. et al. Assembly and role of pili in group B streptococci. Mol. Microbiol. 60, 1404-1413 (2006) FURTHER READING Telford, J. L. et al. Pili in Gram-positive pathogens Nature Rev. Microbiol. 4, 509-519 (2006)

WEBSITE

Patrick Trieu-Cuot's homepage: http://www. pasteur.fr/recherche/departements/DepMifond/ gramplus.html

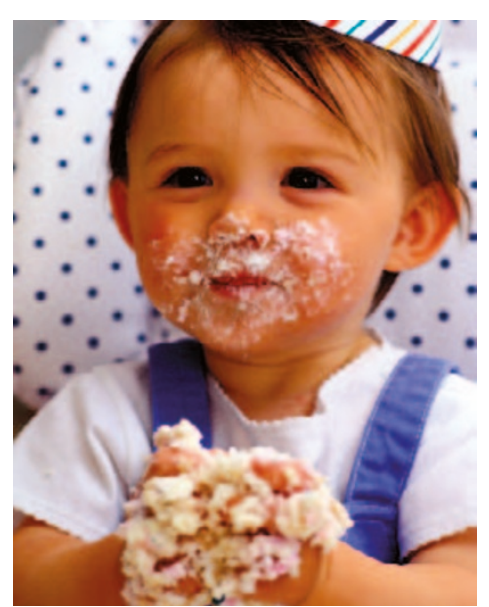

